\title{
Custos e viabilidade econômica na produção de carvão vegetal no estado de Mato Grosso do Sul
}

\author{
Isabela Braga Belchior ${ }^{1^{*}}$, Omar Jorge Sabbag ${ }^{2}$, Deisy Micaelli Sousa Santos Polini ${ }^{1}$, Vitor Gabriel \\ Marinho de Faria Pereira ${ }^{1}$, Arthur Pacheco ${ }^{1}$
}

\begin{abstract}
${ }^{1}$ Departamento de Produção Vegetal, Universidade Estadual de Mato Grosso do Sul (UEMS), Aquidauana-MS, Brasil. ${ }^{2}$ Departamento de Fitotecnia, Tecnologia de Alimentos e Socioeconomia (DFTASE), Universidade Estadual Paulista Júlio de Mesquita Filho (UNESP), Ilha Solteira-SP, Brasil.
\end{abstract}

\begin{abstract}
RESUMO O carvão vegetal é um recurso natural renovável e pode ser usado como agente redutor em siderúrgicas na fabricação de ferro tipo gusa e como fonte de energia. Este trabalho teve como objetivo analisar os custos e da viabilidade econômica para produção de carvão vegetal proveniente de florestas nativas no estado de Mato Grosso do Sul, em um sistema produtivo de fornos do tipo "rabo-quente". O estudo foi realizado em uma carvoaria localizada no município de Nioaque - MS. No cálculo do custo operacional foi adotada a estrutura utilizada pelo Instituto de Economia Agrícola de São Paulo, agrupando-os em: Custo Operacional Efetivo (COE), Custo Operacional Total (COT) e Custo Total de Produção (CTP). Na análise da viabilidade econômica do investimento, foram determinados: Valor Presente Líquido (VPL), Taxa Interna de Retorno (TIR), Razão Benefício-Custo (B/C) e Período de Recuperação do Capital inicial investido (PRC). Observou-se que aproximadamente 87,4\% do COE era composto por despesas com insumos. A análise de rentabilidade demonstrou uma receita líquida de $\mathrm{R} \$ 748.077,38$, com lucratividade de $65,5 \%$. Em relação a viabilidade econômica, o produtor conseguiu recuperar o investimento a partir do $2^{\circ}$ ano de produção. O estudo evidencia a importância de analisar economicamente os empreendimentos de produção de carvão vegetal, e demonstra que a produção de carvão vegetal no sistema produtivo de forno tipo "rabo quente" é viável economicamente.
\end{abstract}

Palavras-chave: gestão; indicadores econômicos; "rabo-quente"; rentabilidade.

\section{Costs and economic viability in charcoal production in the state of Mato Grosso do Sul}

\begin{abstract}
Charcoal is a renewable natural resource and can be used as a reducing agent in the steel making pig iron and a source of energy. This study aimed to analyze the costs and economic viability for the production of charcoal as of native forests in Mato Grosso do Sul, in the productive system of kilns "rabo quente". The study was performed in a charcoal localized in municipality of Nioaque - MS. In the calculate the operating cost was used the model proposed by the Instituto de Economia Agrícola from São Paulo, grouping in: Effective Operating Cost (EOC), Total Operating Cost (TOC) and Total Cost of Production (TCP). In analyzing the economic viability of investment, were determined: Net Present Value (NPV), Internal Rate of Return (IRR), Benefit - Cost Reason (B/C), and Pay Back Period. It was observed that expenses of inputs composed approximately $87.4 \%$ of the COE. Profitability analysis showed a net income of R $\$ 748077.38$, with profitability of $65.5 \%$. In relation to economic viability, the farmer can recover the investment from the 2 rd year of production. The study highlights the importance of economic analysis of the charcoal production enterprises, and demonstrates that the production of charcoal in the productive system of kilns "rabo quente" is economically viable.
\end{abstract}

Keywords: management; economic indicators; "rabo-quente"; profitability. 


\section{Introdução}

A crescente demanda energética, associada à preocupação ambiental com a escassez dos recursos naturais, vem evidenciando a importância de fontes renováveis de energia. Dentre elas, a madeira pode ser utilizada como uma das principais alternativas ao fornecimento de insumos para a produção (LUCENA et al., 2011), podendo assim assegurar o abastecimento energético aliado a manutenção dos recursos naturais.

O carvão vegetal é um recurso natural renovável, menos poluente quando comparado ao carvão mineral e praticamente isento de enxofre e fósforo (ABRAF, 2013). Este apresenta grande relevância na economia brasileira, em especial por sua função como agente redutor em siderúrgicas, transformando o minério de ferro em ferro-gusa, ligas metálicas e aço (OLIVEIRA et al., 2015) demonstrando no ano de 2014 uma participação de 1,5\% no Produto Interno Bruto (PIB) brasileiro e 5,6\% do PIB da Indústria (SGM, 2015).

Segundo Rezende; Santos (2010), o Brasil desde o século XIX é o maior produtor mundial de carvão vegetal, contribuindo diretamente para a siderurgia e em menor escala para o consumo comercial e residencial, sendo os fins siderúrgicos o segundo maior setor de base florestal do país (ABRAF, 2013).

Os principais polos produtores de carvão estão localizados em Minas Gerais, Maranhão, Pará, Espírito Santo e Mato Grosso do Sul (o mais novo polo guseiro), destacando-se também pelo consumo de carvão vegetal para suprir as siderúrgicas instaladas nessas regiões (REZENDE; SANTOS, 2010; VITAL; PINTO, 2009).

No país, os principais sistemas produtivos de carvão vegetal são representados por fornos de alvenaria do tipo "rabo quente", tipo colmeia e os fornos retangulares, além dos fornos metálicos, do tipo container e do tipo cilíndrico metálico vertical (REZENDE; OLIVEIRA, 2013). Entretanto, a produção de carvão vegetal em sua maior parte é realizada em fornos do tipo "rabo-quente", onde a carbonização é subjetiva e controlada por vários fatores, tais como coloração da fumaça, a temperatura das paredes do forno, sentida pelo tato e na observação da aparência dos controladores ar (CARNEIRO et al., 2013).

O rendimento em massa, ou gravimétrico, e a qualidade de carvão vegetal estão associados com as características da madeira e com o modo de condução da carbonização (DAMÁSIO et al., 2015) assim, no sistema de fornos tipo "raboquente" o rendimento em massa está na faixa de $25 \%$, significando grande perda econômica e subutilização da lenha enfornada (PINHEIRO et al., 2006). Entretanto, Brito (1990) afirma que no processo de carbonização aproveitam-se de 30 a $40 \%$ da madeira na forma de carvão vegetal.

Apesar da representatividade do Brasil como maior produtor mundial de carvão vegetal, ainda há pouco desenvolvimento e adoção de novas tecnologias, sendo que $80 \%$ da produção total de carvão permanece em fornos rudimentares, de baixa capacidade volumétrica (OLIVEIRA et al., 2013), que apresentam baixo controle operacional e baixo rendimento em massa, além de emissões não controladas de gases, produzidos pela carbonização, no ambiente (CARVALHO et al., 2012).

Assim, tornam-se necessárias análises econômicas sobre os processos produtivos de produção de carvão vegetal, para um maior incentivo à produção, pois o carvão vegetal é uma eficaz alternativa energética, tanto para a produção siderúrgica quanto para diversos outros segmentos industriais.

A avaliação econômica de um investimento abrange a utilização de critérios e técnicas de análise para comparar os custos e as receitas relacionados ao projeto, objetivando decidir sobre sua implementação ou não (REZENDE; OLIVEIRA, 2013). Sendo assim, projetos executados sem as devidas análises econômicas, como o conhecimento e análise dos custos de operação, podem constituir-se num caminho para o fracasso (CASACA; TOMAZELLI JÚNIOR, 2001). 
Ainda neste sentido, métodos de valoração ambiental são tentativas de traduzir os efeitos dos impactos antrópicos sobre a biodiversidade, capacitando os indivíduos a tomarem decisões economicamente comparáveis.

Este trabalho teve como objetivo a análise dos custos e da viabilidade econômica da produção de carvão vegetal proveniente de florestas nativas no estado de Mato Grosso do Sul, em um sistema produtivo de fornos do tipo "rabo-quente".

\section{Material e Métodos}

A pesquisa foi realizada em uma carvoaria localizada a $21^{\circ} 03^{\prime}$ S e $55^{\circ} 47^{\prime} \mathrm{W}$, no município de Nioaque - MS, que tem o sistema de produção de carvão vegetal através do uso de fornos do tipo "rabo quente".

Este tipo de forno é considerado rudimentar, porém, de baixo custo e de fácil construção. Os fornos são construídos de alvenaria, sendo os tijolos assentados com barro, em forma semiesférica. Em torno do forno são deixadas aberturas para a entrada de ar e saída de gases produzidos pela carbonização, a qual é avaliada e controlada pela experiência do carbonizador.

Na produção de carvão vegetal é utilizada lenha de floresta nativa, a partir realização de contrato de exploração de lenha entre o produtor de carvão e o proprietário rural, onde o produtor de carvão custeia as licenças de regularização do uso da terra e ambientais, como o georreferenciamento, supressão vegetal e outras, totalizando em torno de $\mathrm{R} \$ 15.000,00$.

A carvoaria possui 25 funcionários, cumprindo turno de trabalho de 6 horas diárias e 21 dias úteis por mês, com salário mensal de $\mathrm{R} \$ 1.600,00$ por funcionário, sendo disponibilizada alimentação 3 vezes ao dia e equipamentos de proteção individual adequado para cada função. Além disso, a carvoaria dispõe de poço para captação de água e alojamento de alvenaria para os funcionários.
Os custos para construção de um forno tipo "rabo quente" são em média $R \$ 1.300,00$, os quais possuem vida útil de aproximadamente 1 ano, necessitando assim de manutenção ao final de sua vida útil, a qual é em média $\mathrm{R} \$ 650,00$ por forno. A carvoaria possui 50 fornos, com capacidade de consumo de material lenhoso de $10 \mathrm{~m}^{3} /$ forno em cada carbonização, totalizando 4 carbonizações mensais.

A área de floresta nativa necessária para abastecer os 50 fornos mensalmente é de 17,37 hectares, sendo obtida dividindo o volume médio de madeira consumida por forno por mês $\left(40 \mathrm{~m}^{3}\right)$ pelo volume de $115,1 \mathrm{~m}^{3} / \mathrm{ha}$ de estoque madeireiro presente na área. A extração do material lenhoso é terceirizada, com um custo médio de $\mathrm{R} \$ 500,00$ por hectare.

O carvão vegetal produzido é escoado para uma siderúrgica localizada no município de Aquidauana - MS, distante cerca de $100 \mathrm{~km}$ da carvoaria, com frete no valor de $\mathrm{R} \$ 13,00$ por metro de carvão (mdc).

Para o cálculo de custo de produção foi utilizou-se o modelo de custo operacional adotado pelo Instituto de Economia Agrícola (IEA), proposto por Matsunaga et al. (1976), sendo os custos agrupados em Custo Operacional Efetivo (COE), correspondente aos custos variáveis, como as operações mecanizadas, operações manuais e insumos, somando as despesas gerais e depreciações obtém-se o Custo Operacional Total (COT), bem como o Custo Total de Produção (CTP), obtido acrescentando ao COT a remuneração da terra.

Os indicadores de lucratividade utilizados no trabalho foram os considerados por Martin et al. (1997), sendo: receita bruta, obtida pelo fator multiplicativo da quantidade produzida e o preço recebido pelo produtor; resultado operacional, pela diferença entre a receita bruta e o custo operacional total; e o índice de lucratividade, correspondente a receita líquida dividida pela receita bruta, expresso em porcentagem. Foi verificado ainda o ponto de nivelamento, referente ao mínimo que se deve produzir (em quantidade) para cobrir os custos 
com a produção, bem como ao preço de equilíbrio, resultante do custo total de produção em relação a produtividade obtida.

Para a análise da viabilidade econômica do investimento foi montado um fluxo de caixa, refletindo os valores das entradas e saídas dos recursos e produtos, utilizando um valor de $\mathrm{R} \$ 112,00$ por mdc, preço médio pago pela siderúrgica em agosto de 2015, para um horizonte de planejamento de 4 anos (período de vigência do licenciamento ambiental).

Sendo calculada a Taxa Interna de Retorno (TIR), que representa a taxa anual de retorno do capital investido no projeto, e também pode ser entendida com a taxa de desconto que iguala o valor presente dos custos ao valor presente das receitas (REZENDE; OLIVEIRA, 2013), tendo viabilidade o projeto se a TIR for maior que a Taxa Mínima de Atratividade (TMA), que neste caso, foi utilizada a taxa usual de desconto de $6 \%$ ao ano (referência poupança), expressa por (Equação 1):

$$
\mathrm{VPL}=\sum_{\mathrm{t}=0}^{\mathrm{n}} \frac{\mathrm{FC}_{\mathrm{t}}}{(1+\mathrm{i})^{\mathrm{t}}}=0
$$

Em que: i é a Taxa Interna de Retorno (TIR), $\mathrm{FC}_{\mathrm{t}}$ fluxo de caixa líquido de um determinado período, $\mathrm{n}$ são os números de fluxos, e $\mathrm{t}$ os períodos de produção $(\mathrm{t}=0$ a $\mathrm{t}=\mathrm{n})$.

Foi determinado também o Valor Presente Líquido (VPL), definido como o valor presente dos fluxos de caixa menos o valor inicial do investimento, o qual representa que, se o valor presente das entradas de caixa for, no mínimo, igual ao valor presente das saídas de caixa, então o investimento é viável (SABBAG et al., 2013), e quanto maior o valor do VPL, mais atrativo se torna o projeto, obtido por (Equação 2):

$$
\mathrm{VPL}=\sum_{\mathrm{t}=0}^{\mathrm{n}} \frac{\mathrm{FC}_{\mathrm{t}}}{(1+\mathrm{i})^{\mathrm{t}}}
$$

Determinou-se também o Período de Recuperação do Capital inicial investido (PRC), e a relação Benefício-Custo (B/C), razão obtida entre os valores positivos e negativos do fluxo de caixa (SABBAG et al., 2013), calculada por (Equação 3):

$$
B / C=\sum_{j=0}^{n} \frac{\sum_{j=0}^{n} R j(1+i)^{-j}}{\sum_{j=0}^{n} C j(1+i)^{-j}}
$$

Em que:: $\mathrm{j}$ é período de ocorrência dos custos/receitas, $\mathrm{Rj}$ as receitas no período $\mathrm{j}, \mathrm{Cj}$ os custos no período $\mathrm{j}$, $\mathrm{i}$ a taxa de desconto ou taxa mínima de atratividade e n o número total de anos do fluxo de caixa.

Para complementar as análises, como forma de valoração ambiental, foi calculado o custo de oportunidade, que é a oportunidade renunciada, ou seja, o custo provocado pelo não uso de um recurso (SAMUELSON; NORDHAUS, 2010). Assim, a produção e o consumo se deparam com o dilema entre conservar o meio ambiente ou utilizar os recursos naturais para produzir bens e serviços para uso humano (CALLAN; THOMAS, 2010).

O custo de oportunidade foi obtido pelo lucro líquido por hectare no ano base de análise, multiplicado pela quantidade de hectare preservado dentro da propriedade e descontado de uma taxa de risco do negócio. O desconto de uma taxa de risco deve-se ao fato de não estar produzindo na área preservada; desta forma, ele não estará correndo o risco inerente ao negócio em que está inserido (SANTOS, 2013).

A taxa de risco (y) foi estimada calculando-se o coeficiente de variação da produção de carvão vegetal, utilizando os dados de preços disponíveis no relatório do Ibá (2014) em cada ano de 2006 a 2013. Assim, o custo de oportunidade (C.O.) e o total (C.O. total) foi calculado conforme metodologia proposta por Santos (2013), de acordo com as equações:

C.O. $=$ Lucro líquido por ha ${ }^{\star}$ quantidade de ha preservado 


$$
\text { C.O. total }=\frac{\text { C.O. }}{(1+\mathrm{y})}
$$

\section{Resultados e Discussão}

O investimento total necessário para a instalação de uma carvoaria no município de Nioaque-MS é de R\$ $451.600,00$, sendo as maiores despesas com a aquisição de tratores $(59,8 \%)$, construção dos fornos $(14,4 \%)$ e construção de alojamento (13,3\%), além de despesas com aquisição de insumos.

Tabela 1. Estimativas do Custo Operacional Efetivo (COE), do Custo Operacional Total (COT) e do Custo Total de Produção (CTP) para produção de carvão vegetal no sistema de fornos tipo "rabo quente", 2015.

Table 1. Estimates of the Effective Operating Cost (EOC), Total Operating Cost (TOC) and Total Cost of Production (TCP) for charcoal production in system of kilns type "rabo-quente", 2015.

\begin{tabular}{|c|c|c|c|c|}
\hline Descrição & Unidade & Quantidade & Valor Unitário (R\$) & Valor Total (R\$) \\
\hline Mão de obra & mês & 25 & $1.600,00$ & $40.000,00$ \\
\hline Alimentação & $3 \mathrm{x} / \mathrm{dia}$ & 25 & 8,50 & $4.462,50$ \\
\hline Trator & & 3 & $90.000,00$ & $270.000,00$ \\
\hline Motosserra & & 9 & $2.500,00$ & $22.500,00$ \\
\hline \multicolumn{5}{|l|}{ Insumos } \\
\hline Óleo diesel & $\mathrm{L} / \mathrm{mês}$ & 2500 & 3,20 & $8.000,00$ \\
\hline Combustível & L/mês & 500 & 3,68 & $1.840,00$ \\
\hline EPI's & & 25 & 284,00 & $7.100,00$ \\
\hline Custo Operacional Efetivo (COE) & & & & $353.902,50$ \\
\hline Depreciação Linear (equipamentos) & & & & $22.725,00$ \\
\hline Despesas gerais ${ }^{1}$ & & & & $17.695,13$ \\
\hline Custo Operacional Total (COT) & & & & $394.322,63$ \\
\hline Remuneração da terra & & & & $\mathbf{0 , 0 0}$ \\
\hline Custo Total de Produção (CTP) & & & & $394.322,63$ \\
\hline
\end{tabular}

${ }^{1}$ Refere-se a 5\% do COE.

Fonte: dados da pesquisa.

Tabela 2. Estimativa de custo de oportunidade para a manutenção da área de mata nativa.

Table 2. Estimation of opportunity cost for the maintenance of native forest area.

\begin{tabular}{lr}
\hline Descrição & Valor (R\$/ano) \\
\hline Lucro líquido & $748.077,38$ \\
Área nativa utilizada: 17,37 ha/mês & 208,44 \\
Lucro líquido/ha & $3.588,93$ \\
Custo de oportunidade & $748.077,38$ \\
Taxa de risco (y) & 0,1645 \\
\hline Custo de oportunidade total & $642.403,65$ \\
\hline
\end{tabular}

Fonte: dados da pesquisa.
Os custos operacionais de produção de carvão vegetal estão apresentados na Tabela 1, em que as principais despesas que compõe o custo operacional efetivo foram relacionadas os insumos, os quais representaram $87,4 \%$ do COE.

A depreciação dos equipamentos, por ser caracterizada como custo fixo (Tabela 1) não é considerado um dispêndio monetário, entretanto, deve ser analisada por representar a desvalorização dos equipamentos utilizados. 
Além da depreção, as despesas gerais acrescidas ao COE totalizam o COT, que foi de $R \$ 394.322,63$, sendo $R \$$ $353.902,50$ correspondente ao valor do COE, R $\$ 22.725,00$ a depreciação e $\mathrm{R} \$ 17.695,13$ as despesas gerais.

O CTP correspondeu ao mesmo valor do COT, pois o produtor de carvão não possui remuneração da terra, sendo a exploração de lenha realizada por contrato de exploração.

Verifica-se na Tabela 3 que a receita bruta foi de $R \$$ 1.142.400,00, obtido pela produtividade anual $\left(10.200 \mathrm{~m}^{3}\right) \mathrm{em}$ relação ao preço obtido na comercialização ( $R$ \$112,00/mdc).

A receita líquida foi de $\mathrm{R} \$ 748.077,38$, obtida pela diferença entre a receita bruta pelo o custo total de produção. $\mathrm{O}$ índice de lucratividade (IL) para a atividade foi de $65,5 \%$, que constitui o lucro após a cobertura dos custos, indicando que o sistema de produção de carvão vegetal é rentável, gerando lucros ao produtor.

Para que o produtor consiga cobrir os custos totais de produção (ponto de equilíbrio) é necessário que este produza, no mínimo, $3.520,74 \mathrm{~m}^{3}$ anualmente e venda o carvão produzido a $\mathrm{R} \$ 38,66$, no mínimo.

Considerando-se as possíveis externalidades, sejam negativas ou positivas, na valoração ambiental, as ações antrópicas alteram a emergia original de maneira particular em cada tipo de bioma. Neste sentido, com a valoração ambiental pode-se viabilizar o trade-off entre crescimento econômico e meio ambiente.

De acordo com o CIORD (2012), para representar o cálculo do custo de oportunidade da conservação, utiliza-se a seguinte equação:

$$
\mathrm{CE}=(\mathrm{Rs}-\mathrm{Cs})+\mathrm{Cp}
$$

Em que: CE representa o valor do custo total de uma área de conservação, Rs é o valor da receita bruta calculado pela atividade econômica (carvão); Cs são os custos de produção da atividade e o $\mathrm{Cp}$, referente ao custo associado às ações para proteção ambiental, ou seja, representado pelo custo de oportunidade de manutenção da área preservada.

Assim, o custo da área de conservação de 208 hectares representaria um montante de $\mathrm{R} \$ 1.390 .481,02$, resultante da expressão $C E=(1.142 .400,00-394.322,63)+642.403,65$; superior em $34,1 \%$ à somatória dos custos de produção/manutenção da área correspondente à atividade econômica, inferindo que os custos de conservação se mostram superiores comparativamente à uma área de produção de carvão.

Segundo Maia (2004), embora a proteção ambiental seja desejável do ponto de vista social, a preservação implica num custo que deve ser mensurado para permitir a divisão entre

Tabela 3. Rentabilidade para produção de carvão vegetal em sistema de fornos tipo "rabo quente", 2015.

Table 3. Profitability for charcoal production in system of kilns type "rabo-quente", 2015.

\begin{tabular}{lr}
\hline \multicolumn{1}{c}{ Item } & Valor \\
\hline Preço/mdc & 112 \\
Produção $\left(\mathrm{m}^{3}\right)$ & 10.200 \\
Receita Bruta $(\mathrm{R} \$)$ & $1.142 .400,00$ \\
Custo Total de Produção (R\$) & $394.322,63$ \\
Receita Líquida $(\mathrm{R} \$)$ & $748.077,38$ \\
Índice de Lucratividade (\%) & 65,5 \\
Ponto de equilíbrio - quantidade $\left(\mathrm{m}^{3}\right)$ & $3.520,74$ \\
Ponto de equilíbrio - preço $(\mathrm{R} \$ / \mathrm{mdc})$ & 38,66 \\
\hline
\end{tabular}

Fonte: dados da pesquisa.

os diversos agentes que usufruem dos benefícios da conservação. 
De acordo com Santos (2013), na situação em que o valor da preservação do bioma (VPB) for inferior ao custo de oportunidade, sugere-se ao agente poder optar por explorar comercialmente no campo de atuação da empresa.

Segundo pesquisa do autor, para o custo de oportunidade já calculado $(\mathrm{R} \$ 642.403,65)$ e considerando a relação do valor do bioma total cerrado para Mato Grosso do Sul (VBT) em relação à sua área total de $\mathrm{R} \$ 269,25 /$ ha e área a ser preservada com a atividade (208 ha), além do fator $1 / 1+\Delta \mathrm{p}$ (ou fator $\mathrm{K}=1,09$, considerado este como a média das principais culturas de importância econômica para o cerrado) e área remanescente em cerrado (104.184.679,68 ha), conforme métrica de Valoração Ambiental proposta abaixo:

$$
\mathrm{VPB}=\frac{\mathrm{C} . \mathrm{O} .}{(1+\mathrm{y})}+\left(\left(\frac{\mathrm{VBT}}{\text { ha total }} \text { ha preserv. }\right)^{*}\left(1+\frac{\text { ha preserv. }}{\text { ha reman. }}\right)\right) * \frac{1}{1+\Delta \mathrm{p}}
$$

O VBP resultou em $\mathrm{R} \$ 703.446,70$, valor inferior ao custo de oportunidade da atividade carvoeira, em R $\$ 748.077,38$, reforçando com isto uma possível concessão ao agente preservador um certificado passível de troca e comercialização, sendo o custo de oportunidade de sua preservação dado pelos benefícios de uma possível atividade de exploração de madeira, como sugere o trabalho em questão. Assim, o custo de oportunidade representa a produção de carvão que poderia estar sendo desenvolvida na área de proteção em virtude das supostas restrições ambientais.

Finalmente, os indicadores de avaliação da viabilidade econômica estão apresentados na Tabela 4. A análise do investimento para o horizonte de planejamento de 4 anos demonstrou VPL positivo, de R\$175.687,64, indicando que há viabilidade econômica nesse sistema produtivo, corroborando com Oliveira et al. (2014), que também constataram VPL positivo para fornos do tipo "rabo quente".

A TIR foi de $22 \%$, superior à taxa mínima de atratividade, sendo que quando a taxa interna de retorno superar taxa de desconto correspondente à taxa de remuneração (TMA) as perspectivas são de que se obtenha mais ganho em investir na atividade, indicando que o projeto é considerado viável economicamente (KREUZ et al., 2008; SAMPAIO et al., 2007).

A relação B/C foi superior a 1, demonstrando viabilidade econômica da produção. A razão encontrada foi de 1,06, indicando um retorno de $6 \%$ para o produtor na atividade. Porém, Oliveira et al. (2014) encontram um índice de 1,21, em sistema de condução de brotação de cepas, esse maior valor pode ser devido aos menores custos com insumos.

Em relação ao período necessário para recuperar o investimento inicial (PRC), para esse sistema de produção foi de 2,49 anos, tempo semelhante ao encontrado por Silva el al. (2014), que obtiveram um PRC de 2,84 anos para fornos tipo "rabo quente".

Tabela 4. Indicadores de viabilidade econômica para produção de carvão vegetal em sistema de fornos tipo "rabo quente", 2015. Table 4. Economic viability indicators for charcoal production in system of kilns type "rabo-quente", 2015.

\begin{tabular}{lc}
\hline Índice & Valor \\
\hline Relação B/C & 1,06 \\
Taxa de desconto \% a.a. & $6 \%$ \\
VPL & $175.687,64$ \\
TIR & $22 \%$ \\
PRC & 2,49 anos \\
\hline
\end{tabular}

Fonte: dados da pesquisa. 
Diante dos resultados positivos encontrados através da avaliação dos indicadores econômicos para produção de carvão vegetal em fornos do tipo "rabo quente", pode-se perceber que o investimento é um bom negócio e apresenta resultados econômicos atrativos.

\section{Conclusões}

Este estudo salienta a relevância de analisar economicamente os empreendimentos de produção de carvão vegetal, pois possibilita auxiliar na tomada de decisão sobre o investimento com base na viabilidade do projeto.

Um dos métodos de valoração ambiental aplicado permitiu refletir sobre o valor do meio ambiente e a pertinência de desenvolver atividades econômicas que está à disposição e sob a responsabilidade das empresas.

Apesar da produção de carvão vegetal ser realizada de maneira rudimentar e com baixo rendimento gravimétrico, esta atividade se demonstra atrativa e viável economicamente.

\section{Referências}

ASSOCIAÇÃO BRASILEIRA DE PRODUTORES DE FLORESTAS PLANTADAS - ABRAF. Anuário estatístico da ABRAF: ano base 2012. Brasília, 2013. 148 p.

BRITO, J. O. Princípios de produção e utilização de carvão vegetal de madeira. Documentos florestais, v. 9, p. 1-19. 1990.

CALLAN, S. J.; THOMAS, J. M. Environmental economics \& management: theory, policy, and applications. Mason, OH: South-Western, Cengage Learning, 2010. 588p.

CARNEIRO, A. C. O.; VITAL, B. R.; OLIVEIRA, A.C.; PEREIRA, B. L. C. Pirólise lenta da madeira para produção de carvão vegetal. In: SANTOS, F.; COLLODETTE, J.; QUEIROZ, J.H. Bioenergia \& Biorrefinaria - Cana-deAçúcar \& Espécies Florestais. Viçosa: UFV, 2013. p. 429-457.

CARVALHO, S. R.; BORGESI, V. L.; B. H. O.; OLIVEIRA, R. L. M.; FIGUEIRA JÚNIOR, E. A.; PESSOA FILHO, J. S. Instrumentação térmica aplicada ao processo de produção de carvão vegetal em fornos de alvenaria. Revista Árvore, Viçosa, v. 36, n. 4, p. 787-796. 2012.

CASACA, J. M.; TOMAZELLI JÚNIOR, O. Planilhas para cálculos de custo de produção de peixes. Florianópolis: Epagri, 2001. 38p. (Documentos, 206).

CENTRO INTEGRADO DE ORDENAMENTO TERRITORIAL - CIORD. Métodos de valoração ambiental: método de custo de oportunidade. 2012. Disponível em $<$ http://www.icmbio.gov.br/ead/file.php/1/paginas/inscricoes/2012/arquivos/13.ICMBIO_Va-

1Amb_II_Maio2012.pdf>. Acesso em: 04 Julho 2017.

DAMÁSIO, R. A. P.; OLIVEIRA, A. C.; CARNEIRO, A.C. O.; BARCELOS, D. C.; PEREIRA, B. L. C.; MAGALHAES, M. A. SILVA, C. M. S. Perfil térmico e controle da carbonização em forno circular por meio da temperatura interna. Ciência da Madeira, v. 6, n. 1, p. 11-22. 2015.

INDÚSTRIA BRASILEIRA DE ÁRVORES - IBÁ. Relatório IBÁ 2016. Brasília: Pöyry, 2014, 100p.

KREUZ, C. L; SOUZA, A; CLEMENTE, A. Custos de produção, expectativas de retorno e de riscos do agronegócio mel no planalto norte de Santa Catarina. Custos e @gronegócio on line, Recife, v. 4, n. 1, p. 46-61. 2008.

LUCENA, L. P.; KLIEMANN NETO, F. J.; MASSUIA, F. M.; FREITAS, C. E. A demanda por carvão vegetal e suas consequências econômicas sobre o agronegócio do eucalipto no Brasil. Revista de Estudos Sociais, Mato Grosso, v. 13, n. 25, p. 158-172. 2011.

MAIA, A. G, ROMEIRO, A. R; REYDON, B. P. Valoração de recursos ambientais: metodologias e recomendações. Campinas: Unicamp. Instituto de Economia, mar. 2004. 38 p.

MARTIN, N. B; SERRA, R; OLIVEIRA, M.D.M; ÂNGELO, J.A; OKAWA, Hl. Sistema "CUSTAGRI": sistema integrado de custos agropecuários. São Paulo: IEA/SAA, 1997. 75 p.

MATSUNAGA, M.; BEMELMANS, P. F.; TOLEDO, P. E. N. Metodologia de custo utilizada pelo IEA. Agricultura em São Paulo, São Paulo, v. 23, n. 1, p. 123-139. 1976.

OLIVEIRA, A. C.; CARNEIRO, A. C. O.; BARCELLOS, D. C.; RODRIGUEZ, A. V.; AMARAL, B. M. N.; PEREIRA, B. L. C. Resfriamento artificial em fornos retangulares para a produção de carvão vegetal. Revista Árvore, Viçosa, v. 39, n. 4, p. 769-778. 2015. 
OLIVEIRA, A. C.; CARNEIRO, A. C. O.; PEREIRA, B. L. C.; VITAL, B. R.; CARVALHO, A. M. L.; TRUGILHO, P. F.; DAMÁSIO, R. A. P. Otimização da produção do carvão vegetal por meio do controle de temperaturas de carbonização. Revista Árvore, Viçosa, v. 37, n. 3, p. 557-566. 2013.

OLIVEIRA, A. C.; SALLES, T. T.; PEREIRA, B. L. C.; CARNEIRO, A. C. O.; BRAGA, C. S.; SANTOS, R. C. Viabilidade econômica da produção de carvão vegetal em dois sistemas produtivos. Floresta, Curitiba, v. 44, n. 1, p. 143 - 152. 2014.

PINHEIRO, P. C. C.; REZENDE, M. E. A.; SAMPAIO, R. S. A produção de carvão vegetal: teoria e prática. Belo Horizonte, 2006. 120 p.

REZENDE, J. B.; SANTOS, A. C. D. A cadeia produtiva do carvão vegetal em Minas Gerais: pontos críticos e potencialidades. Viçosa, MG: EPAMIG, 2010. 82 p. (Boletim Técnico, 95).

REZENDE, J. L. P.; OLIVEIRA, A. D. Análise econômica e social de projetos florestais. 3. ed. Viçosa: UFV, 2013. 385 p.

SABBAG, O. J.; NICODEMO, D.; OLIVEIRA, J. E. M. Custos e viabilidade econômica da produção de casulos do bicho-daseda. Pesquisa Agropecuária Tropical, Goiânia, v. 43, n. 2, p. 187-194. 2013.

SAMPAIO, L. C.; NETO, S. N. O.; LELES, P. S. S.; SILVA, J. A.; VILLA, E. B. Análise técnica e econômica da produção de palmito de pupunha (Bactris gasipaes Kunth.) e de palmeirareal. Floresta e Ambiente, Rio de janeiro, v. 14, n. 1, p. 14-24. 2007.

SECRETARIA DE GEOLOGIA, MINERAÇÃO E TRANSFORMAÇÃO MINERAL - SGM. Anuário Estatístico: Setor Metalúrgico. Brasília: 2015. 108 p.

SAMUELSON, P. A.; NORDHAUS, W.D. Economia. Madrid: McGraw-Hill, 2005.

SANTOS, L. J. M. Proposta de métrica de valoração ambiental para reservas legais e áreas de preservação permanente. USP, Ribeirão Preto, 2013. Dissertação de mestrado em Controladoria e Contabilidade. $333 \mathrm{f}$.

SILVA, D. A. L.; CARDOSO, E. A. C.; VARANDA, L. D.; CHRISTOFORO, A. L.; MALINOVSKI, R. A. Análise de viabilidade econômica de três sistemas produtivos de carvão vegetal por diferentes métodos. Revista Árvore, Viçosa, v. 38, n. 1, p. 185-193. 2014.
VITAL, M. H. F.; PINTO, M. A. C. Condições para a sustentabilidade da produção de carvão vegetal para fabricação de ferro-gusa no Brasil. BNDS setorial, n. 30, p. 237-297. 2009. 